\title{
Thermally Activated Contact Strengthening Explains Nonmonotonic Temperature and Velocity Dependence of Atomic Friction
}

\author{
Mykhaylo Evstigneev* and Peter Reimann \\ Universität Bielefeld, Fakultät für Physik, 33615 Bielefeld, Germany \\ (Received 3 September 2013; revised manuscript received 18 October 2013; published 26 November 2013)

\begin{abstract}
While the well-established Prandtl-Tomlinson (PT) model of atomic friction predicts that the friction force decreases with temperature and grows with velocity, several recent experiments reported that a nonmonotonic temperature dependence and a decreasing velocity dependence may also occur. We propose a minimal extension of the PT model, incorporating the possibility of thermally activated contact strengthening and providing one common framework to quantitatively explain all those "anomalous" experimental findings, as well as the previously known "normal" (PT-like) behavior.
\end{abstract}

DOI: 10.1103/PhysRevX.3.041020

The everyday-life macroscopic friction results from the interactions between large numbers of asperities on the microscopically rough surfaces in contact [1]. To understand the nature of this extremely complex phenomenon, atomic-friction experiments focus on the interaction properties of a single asperity - the tip of an atomic force microscope (AFM) - with an atomically flat surface [2]. It is by now a generally accepted viewpoint that the typical nanoscale stick-slip friction observed in such experiments is a thermally activated process: When the AFM tip is dragged at a constant pulling velocity along an atomically flat substrate, its interstitial transitions are thermally assisted events, governed by a Kramers-Arrhenius rate of the form $\gamma e^{-\Delta U(f) / k T}$, where $f$ is the lateral force, $\Delta U(f)$ the force-dependent energy barrier, $k T$ the thermal energy, and $\gamma$ an approximately constant (i.e., $f$ - and $T$-independent) rate prefactor [2]. This model, which goes back to the pioneering works by Prandtl and Tomlinson [3] and is referred to as the PT model hereafter, predicts that friction should monotonically decrease with temperature and grow with pulling velocity [2].

Surprisingly, recent experimental results obtained by Schirmeisen et al. [4-6] revealed that, for a number of materials tested, namely, $\mathrm{Si}, \mathrm{SiC}, \mathrm{NaCl}$, and highly oriented pyrolytic graphite (HOPG), the mean friction force actually exhibits a maximum as a function of temperature. Moreover, at the lowest temperature probed in Refs. [5,6], friction decreased with pulling velocity. In the same vein, measurements done on HOPG under slightly different experimental conditions [7] also showed a nonmonotonic temperature dependence of friction, although the maximum was not as pronounced as in Refs. [4-6]; and even

\footnotetext{
*mykhaylo@physik.uni-bielefeld.de
}

Published by the American Physical Society under the terms of the Creative Commons Attribution 3.0 License. Further distribution of this work must maintain attribution to the author(s) and the published article's title, journal citation, and DOI.
Subject Areas: Condensed Matter Physics, Nanophysics

more remarkable, this maximum was, in addition, preceded by a minimum.

Since this "anomalous" behavior cannot be explained within the traditional PT model (see above), two very different theoretical Ansätze have recently been put forward to interpret those experimental results from Refs. [4-7].

Within the "mechano-kinetic model" from Refs. [5,6] (see also Sec. IV of the recent review [8]), the tip-substrate interface was assumed to be formed not by a single but rather by many (typically, a few tens of) elastic contacts between binding sites on the AFM tip and the substrate. Formation and rupture of each such contact was assumed to be a thermally activated process occurring with rates proportional to $e^{-\Delta E_{\mathrm{on}} / k T}$ and $e^{-\Delta E_{\mathrm{off}} / k T}$, where $\Delta E_{\mathrm{on}, \text { off }}$ are the respective energy barriers [9]. In the original model from Ref. [5], the tip binding sites represented randomly distributed asperities on the surface of an amorphous AFM tip. A somewhat different physical picture was adopted in the subsequent publication [6]: Namely, the binding sites were associated with the regularly arranged atoms of a nanocrystal, which was assumed to be removed from the $\mathrm{NaCl}$ substrate and rigidly attached to the AFM tip.

The main virtue of those mechano-kinetic models is that they indeed nicely reproduce the experimental observations from Refs. [4-6]. On the other hand, the models adopted both in Ref. [5] and in the main body of Ref. [6] cannot explain the occurrence of a minimum in the friction vs temperature data reported in [7]. In principle, it is conceivable that a generalized model, as outlined at the very end of Ref. [6], may be able to reproduce such a minimum, albeit this has not been shown explicitly so far [10].

An alternative theoretical proposal was put forward in Ref. [7], namely, that the rate prefactor $\gamma$ in the PT model actually depends on temperature and therefore should be considered as a fit parameter for each temperature probed. In this way, all the friction-velocity curves from Ref. [7] could indeed be fitted quite well. However, even with such a temperature-dependent prefactor, the PT model still implies that, for any given temperature, the friction force is 
necessarily an increasing function of velocity. Hence, the opposite behavior observed in Refs. [5,6] cannot be explained in this way. Furthermore, from a theoretical viewpoint, the strong temperature dependence of the rate prefactor $\gamma$ found in Ref. [7], differing by a factor of 24 for $T=155 \mathrm{~K}$ and $T=202 \mathrm{~K}$, is hard to justify in view of the usually very weak temperature dependences of such prefactors in the context of thermally activated rate theory [9].

It should be emphasized that the basic physical picture behind the PT model is quite different from that behind the mechano-kinetic model. The PT model starts from the motion of the AFM tip in a periodic, free-energy-type potential landscape, arising as a potential of mean force due to the fast molecular degrees of freedom at the accompanying thermal equilibrium and reflecting the atomic periodicity of the probed surface. In a next step, this continuous description is approximated by a discrete rate model describing the thermally activated interstitial transitions of the tip over the periodic potential maxima [2]. In contrast, the mechano-kinetic model starts from the picture of an approximately "free sliding motion" of the AFM tip along an essentially "flat" (translationally invariant) surface, only subject to a position-independent and rather weak dissipation, e.g., due to phonons [6]. In a next step, the model is augmented by a rate description of the making and breaking of bonds between AFM tip and substrate at certain randomly or regularly distributed binding sites (see above). In view of those quite significant conceptual differences between the two approaches, it seems unlikely to us that both of them may be considered simultaneously as a faithful description of a given experimental system.

In the following, we propose a unification of both approaches, namely, a minimal extension of the PT model, incorporating the possibility of thermally activated contact strengthening along the lines of the mechano-kinetic model, and providing one common framework to quantitatively explain all those anomalous experimental findings from Refs. [4-7], as well as all the previously known "normal" findings, which could already be described very well by the standard PT model.

Within the realm of macroscopic friction, the concept of contact strengthening was introduced as a phenomenological description of aging processes some 30 years ago [11]. This concept has been further developed, e.g., in [12]. In the field of AFM nanofriction, contact strengthening due to bond formation was proposed to explain the experimental statistics of stick-slip events in Ref. [13]. This idea was microscopically confirmed and refined in Refs. [14,15], unraveling the underlying specific molecular processes in quantitative detail. Further possible aging mechanisms are the formation of capillary bridges in a humid environment [16] and the formation of a neck due to material transfer from the substrate into the contact region with the tip [17]. Finally, recent molecular-dynamics simulations revealed that the AFM tip may, in the course of time, evolve into a different geometrical contact configuration characterized by a different energy of binding to the surface [18].

While aging usually gives rise to a logarithmic growth of the friction force with time [12,14-16] and proceeds via a sequence of transitions between many metastable states of the system, our present model involves only one such transition, which thus may be regarded in some sense as an "elementary aging event." In principle, an arbitrary number of such events can be incorporated into the model, so as to reproduce, with the help of a suitable distribution of transition barrier heights, the above-mentioned characteristic logarithmic signature of aging, similar to the derivations in Refs. $[15,16]$. On the other hand, we found that such an extension of our present minimal model, which inevitably would also increase the number of model parameters, is actually unnecessary to quantitatively explain the experimental findings from Refs. [4-7].

We start from the well-established description of nanoscale friction by AFM in terms of alternating stick phases and interstitial slip events along a spatially periodic, atomically flat substrate [2]. The basic idea of our model is that, in any given stick phase, the tip-sample contact may either break, resulting in a slip event, or strengthen itself by one of the molecular aging mechanisms described above [13-18]. Within our minimal model, we assume just two possible stick states of the AFM tip: a weakly bound state $A$ and a strongly bound state $B$ (see Fig. 1). Initially (right after the preceding quasi-instantaneous slip event), the weakly bound state $A$ is formed. From then on, the tip has two possibilities: (i) to jump by way of an interstitial slip event to the next lattice site and form a contact in state $A$ anew (the rate of this process is denoted as $\omega_{A}$ ); (ii) to strengthen the contact with the substrate by entering a strongly bound state $B$ with a rate $\Omega$ and subsequently jump to the next lattice site with a different rate $\omega_{B}$, forming the state $A$ there anew. More precisely, from any given lattice site, interstitial jumps can occur in either direction, henceforth denoted as forward and backward slip events. Correspondingly, we are actually dealing with two forward rates, $\omega_{A}^{+}$and $\omega_{B}^{+}$, and two backward rates, $\omega_{A}^{-}$and $\omega_{B}^{-}$. While $\omega_{A, B}^{+}$, in general, depend on the acting force $f$ and temperature $T$, we assume as a simplifying approximation that the contact-strengthening rate $\Omega$ depends on temperature only, the rationale being that a contact-strengthening process occurs at one and the same

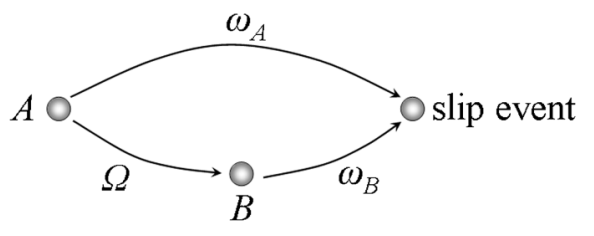

FIG. 1. Basic scheme of the contact-strengthening model from the main text. 
lattice site and thus couples to the lateral force much more weakly than an interstitial jump process. Finally, we assume that, for symmetry reasons, backward jumps at a force value $f$ occur at the same rate as forward jumps at a force value $-f$, i.e.,

$$
\omega_{A, B}^{-}(f, T)=\omega_{A, B}^{+}(-f, T) .
$$

Regarding the quantitative modeling of the rates, we follow the previously developed concepts of the PT model mentioned in the first paragraph [2]. Namely, the transition rates (1) are of the standard Kramers-Arrhenius form [9]

$$
\omega_{A, B}^{+}(f, T)=\gamma_{A, B} e^{-\Delta U_{A, B}(f) / k T},
$$

with approximately $f$ - and $T$-independent rate prefactors $\gamma_{A, B}$. Further, the potential barriers are approximated as [2]

$$
\Delta U_{A, B}(f)=\Delta U_{A, B}^{0}\left[1-f / f_{A, B}\right]^{3 / 2},
$$

where $\Delta U_{A, B}^{0}$ is the barrier height at zero force, and $f_{A, B}$ is the critical force at which the barrier vanishes. Likewise, the rate of contact strengthening is given by

$$
\Omega(T)=\Gamma e^{-\Delta E / k T} .
$$

Because of the externally imposed, constant pulling velocity $v$, the lateral force increases linearly in time within any given stick phase (labeled by an integer "stick-state index" $n$ ) and instantaneously drops with every forward slip event (increment of $n$ by unity), and analogously for backward slips. In other words,

$$
f(t)=\kappa[v t-n(t) a],
$$

where $\kappa$ is the effective lateral spring constant and $a$ the lattice constant of the substrate. The stick-state index $n(t)$ changes by \pm 1 according to the above-specified jump rates $\omega_{A, B}^{ \pm}(f(t), T)$, governing the various slip events. Finally, the mean friction force follows as the longtime average of $f(t)$ and is denoted as $\bar{f}$.

To gain insight into the basic properties of our model, we first focus on fast pulling velocities $v$, such that the mean frequency of interstitial slips, $v / a$, is much larger than the bond-strengthening rate $\Omega(T)$ within any given stick phase. As a consequence, the strongly bound state $B$ is hardly visited, and we recover the original PT model with jump rates $\omega_{A}^{ \pm}(f, T)$, henceforth denoted as the PTA model. Likewise, for $v / a \ll \Omega(T)$, the system spends most of its time in the strongly bound state $B$, thus effectively amounting to a PT model with jump rates $\omega_{B}^{ \pm}(f, T)$ (PTB model). Our basic assumption that the state $B$ is "stickier" than the state $A$ amounts to $\omega_{A}^{ \pm}(f, T)>\omega_{B}^{ \pm}(f, T)$. As expected, this implies [2] that for any given values of $v$ and $T$, the mean friction force $\bar{f}$ of the stickier PTB model always exceeds that of the PTA model. In combination with the well-known behavior of the PT model, namely, that the mean friction force $\bar{f}$ grows with pulling velocity $v$ and decreases with temperature $T$ [2], we thus arrive at the following overall picture: As a function of the pulling velocity $v$, the mean friction force $\bar{f}(v)$ is growing both for very small and for very large $v$ [see Fig. 2(a)]. The crossover between those two asymptotics occurs around $v \approx a \Omega(T)$. If the binding strength of state $B$ sufficiently exceeds that of state $A$, we furthermore expect that the two "growing segments" (for small and large $v$ ) of $\bar{f}(v)$ will exhibit a substantial "offset" and hence will be connected by a decreasing intermediate segment with a local maximum and minimum at its left and right ends, respectively [see Fig. 2(a)]. Similarly, the force-temperature characteristics $\bar{f}(T)$ (at fixed $v$ ) are expected to exhibit a decreasing behavior for large and small $T$, connected by an increasing crossover region for temperatures $T$, which approximately satisfies $\Omega(T) \approx v / a$. All those predictions are confirmed and quantitatively exemplified by Fig. 2 . Note, also, that the "crossover condition" $\Omega(T) \approx v / a$ is, in view of (4), highly "asymmetric" with respect to the quantitative dependence on $v$ and $T$ : It implies a relatively weak dependence of the "increasing segment" of $\bar{f}(T)$ on $v$ [see Fig. 2(b)], while the "decreasing segment" of $\bar{f}(v)$ will quickly migrate even upon relatively small variations of $T$ [see Fig. 2(a)]. For similar reasons, the overall dependence of $\bar{f}$ on $v$ is much more modest [essentially logarithmic; see Fig. 2(a)] than on $T$ [Fig. 2(b)].

It follows that if the parameter range probed does not include the crossover region [i.e., either $\Omega(T) \gg v / a$ or $\Omega(T) \ll v / a$ for all $T$ and $v$, our present model behaves essentially as the original PT model. Moreover, if the binding strengths of $A$ and $B$ hardly differ $\left[\omega_{A}^{ \pm}(f, T) \approx \omega_{B}^{ \pm}(f, T)\right]$, our distinction between the states $A$ and $B$ becomes superfluous, and we recover, once again, the original PT model, but now for arbitrary $v$ and $T$ values.
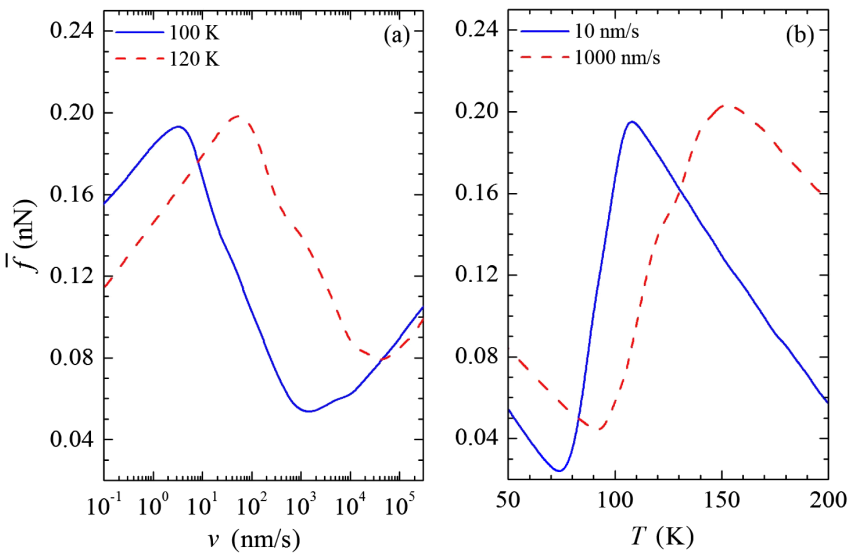

FIG. 2. (a) Mean friction force $\bar{f}$ versus pulling velocity $v$ by numerically solving (1)-(5), as detailed in the main text. Solid blue line: $T=100 \mathrm{~K}$. Dashed red line: $T=120 \mathrm{~K}$. (b) Mean friction force $\bar{f}$ versus temperature $T$ for $v=10 \mathrm{~nm} / \mathrm{s}$ (solid blue line) and $v=1000 \mathrm{~nm} / \mathrm{s}$ (dashed red line). All model parameters are adopted from our fitting to the experimental data from Ref. [6] and are listed in the caption of Fig. 3(b). 
To quantitatively compare our model (1)-(5) with experimental data, we have performed numerical simulations based on a Monte Carlo algorithm [19]. Specifically, we focus on the two above-mentioned experiments from Ref. [7] and from Ref. [6] and fit the model parameters $\gamma_{A, B}$, $\Delta U_{0}^{A, B}, f_{A, B}, \Gamma$, and $\Delta E$ to each of the two experimental data sets ( $\kappa$ and $a$ were directly taken from Refs. [6,7]).

The so-obtained comparison between our model (1)-(5) and the data from Refs. [6,7] is presented in Figs. 3(a) and 3(b), respectively. Overall, the agreement between theory and experiment is obvious. In particular, the simultaneous appearance of a minimum and a maximum in the data from Fig. 3(a) is nicely explained by the theory. A similar behavior of the system from Fig. 3(b) may be considered as a prediction of our theory upon further decreasing the temperature in the corresponding experiment.

The agreement between theory and experiment in Fig. 3(b) is somewhat worse than in Fig. 3(a). In particular, the experimental findings for the two lowest velocities $(v=52 \mathrm{~nm} / \mathrm{s}$ and $v=104 \mathrm{~nm} / \mathrm{s}$ ) in Fig. 3(b) may be considered as indicating the transition from a single to a double peak around $T=150 \mathrm{~K}$. Neither the present nor any previous theory in this context can explain such a feature. On the other hand, it may also be simply an artifact of the experimental uncertainty (see also Fig. 1a in Ref. [6]), and, indeed, this feature has never been seen in any other experiment as far as we know.

We remark that in the experimental work [7], it was not the mean friction forces $\bar{f}$ but rather the so-called
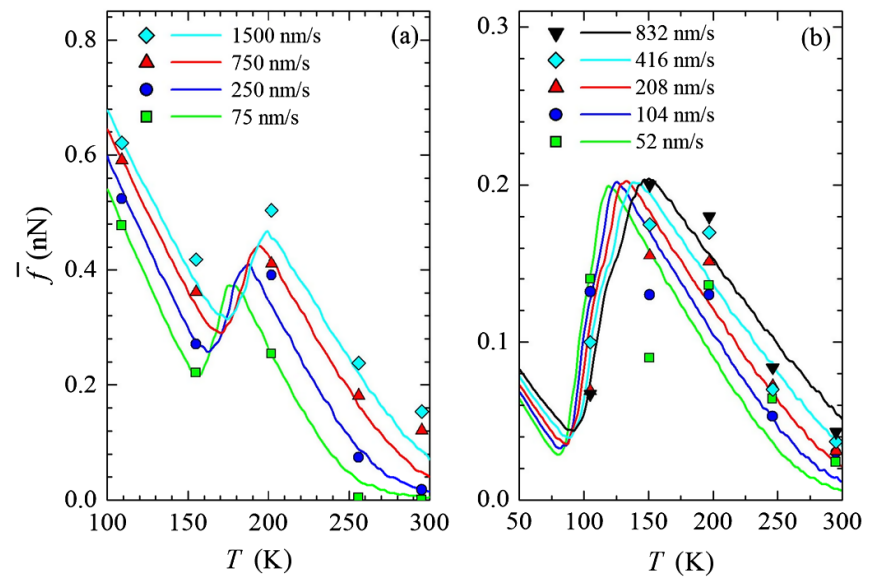

FIG. 3. Mean friction force $\bar{f}$ versus temperature $T$ for various pulling velocities $v$. Symbols: Experimental data extracted from (a) Fig. 4a of Ref. [7] (HOPG substrate) and (b) Fig. 1c of Ref. [6] ( $\mathrm{NaCl}$ substrate). Lines: Numerically obtained solutions of the theoretical model (1)-(5). Parameters in (a): $\gamma_{A, B}=10 \mathrm{GHz}, \Delta U_{0}^{A}=65 \mathrm{pN} \mathrm{nm}, \Delta U_{0}^{B}=80 \mathrm{pNnm}$, $f_{A}=2.121 \mathrm{nN}, \quad f_{B}=2.454 \mathrm{nN}, \quad \Gamma=1000 \mathrm{THz}, \Delta E=$ $67 \mathrm{pN} \mathrm{nm}, \kappa=4.95 \mathrm{~N} / \mathrm{m}$, and $a=0.246 \mathrm{~nm}$. Parameters in (b): $\gamma_{A, B}=0.1 \mathrm{GH} z, \Delta U_{0}^{A}=35 \mathrm{pNnm}, \Delta U_{0}^{B}=62 \mathrm{pN} \mathrm{nm}$, $f_{A}=0.29 \mathrm{nN}, f_{B}=0.59 \mathrm{nN}, \Gamma=50 \mathrm{GH} z, \Delta E=26 \mathrm{pN} \mathrm{nm}$, $\kappa=0.9 \mathrm{~N} / \mathrm{m}$, and $a=0.282 \mathrm{~nm}$. most-probable slip-inducing forces $f^{*}$ that were actually evaluated and reported. The relation between these two forces is very well approximated by [20]

$$
\bar{f}=f^{*}-\kappa a / 2,
$$

provided that backward slip events [see text above Eq. (1)] do not play a significant role. In Fig. 3(a), we have exploited Eq. (6) for reconstructing the mean friction forces $\bar{f}$ from the experimental $f^{*}$ values reported in Ref. [7]. Furthermore, we omitted the experimental results for the lowest pulling velocity $v=50 \mathrm{~nm} / \mathrm{s}$ from Ref. [7] since the theoretical model revealed that backward slip events play a significant role at such a low velocity. Likewise, at high temperatures ( $T=256 \mathrm{~K}$ and $T=295 \mathrm{~K}$ ) backward slip events turned out to be non-negligible even for the second- and third-lowest pulling velocities $(v=75 \mathrm{~nm} / \mathrm{s}$ and $v=125 \mathrm{~nm} / \mathrm{s}$ ) from Ref. [7]. In fact, upon sufficiently magnifying (e.g., on a computer screen) Fig. 3c in Ref. [7], one can see the appearance of such backward slip events already in the experimentally observed stickslip forces for $v=125 \mathrm{~nm} / \mathrm{s}$ and $T=295 \mathrm{~K}$ (even though the experimental data filtering tends to hide such events). In such cases, Eq. (6) may not be a good approximation and, in fact, sometimes even produces negative $\bar{f}$ values, which is clearly unphysical. Rather than simply omitting the corresponding data points, we still employ (6) in Fig. 3(a), except that negative results for $\bar{f}$ are replaced by $\bar{f}=0$. The fact that the "true" experimental $\bar{f}$ values are indeed close to zero in such cases is, once again, confirmed by Fig. 3c from Ref. [7]. Finally, we also omit in Fig. 3(a) the experimental data from Ref. [7] for the intermediate velocities $v=125 \mathrm{~nm} / \mathrm{s}$ and $v=375 \mathrm{~nm} / \mathrm{s}$ in order not to overload the figure. The agreement of those omitted data with the theory is similar to those shown in Fig. 3(a), as can also be inferred from Fig. 4(a), where all the data for $v=50,125$, and $375 \mathrm{~nm} / \mathrm{s}$ are included.
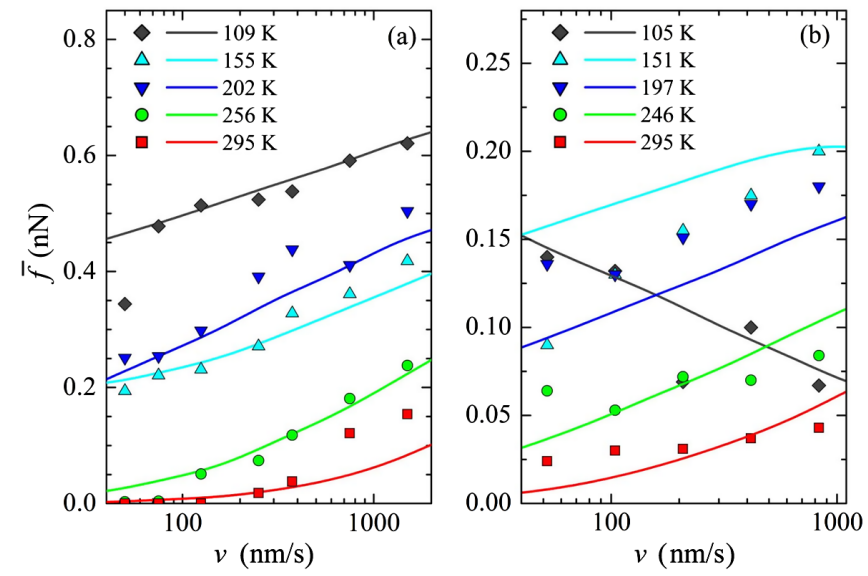

FIG. 4. Mean friction force $\bar{f}$ versus pulling velocity $v$ for various temperatures $T$. Symbols: Data from the same experiments as in Fig. 3. Lines: Theory (1)-(5) with the same parameters as in Fig. 3. 
To draw any further conclusions from the quantitative fit-parameter values obtained in Fig. 3 with respect to the probed $\mathrm{HOPG}$ and $\mathrm{NaCl}$ substrates does not seem possible to us since all those parameter values are also expected to critically depend on the experimentally applied normal load force and on the details of the AFM tip. In particular, the rate prefactors $\gamma_{A, B}$ and $\Gamma$ cannot be directly related to any molecular "oscillation frequencies," since they also depend on the pertinent coupling strength to the thermal environment via some effective dissipation or damping coefficient [9].

In Fig. 4, the very same experimental data from Refs. [6,7] are, once again, compared with our model (1)-(5), but now as $\bar{f}$ versus $v$ curves for various fixed $T$ values [and without omitting any data points in (a)]. The most interesting feature is the decreasing friction curve for $T=105 \mathrm{~K}$ in Fig. 4(b). All other curves in Fig. 4(b) exhibit the usual (PT-like) growth. In particular, we see that the decreasing segment of $\bar{f}(v)$ indeed moves out of the considered $v$ interval very quickly upon variation of $T$, as predicted by the theory [see also Fig. 2(a)]. Except for $T=105 \mathrm{~K}$ in Fig. 4(b), all other curves in Fig. 4 increase essentially logarithmically with $v$, in accordance with the standard PT model and numerous other experimental findings [3]. Only for very low $v$ and high $T$ (where the logarithmic curves would assume negative values) does a crossover to almost zero forces $\bar{f}$ take place. In fact, this crossover behavior is already predicted by the PT model, provided backward slip events are included.

To summarize, aging effects are known to play a key role in the emergence of static friction at the macroscopic scale [12]. Yet, the exploration of aging at the level of singleasperity nanoscale contacts has been initiated only relatively recently [5,6,13-17], while earlier studies of nanofriction invoked the traditional PT model, which assumes unchanging tip-substrate contact properties $[2,7,19,20]$. In this work, we have extended the PT model of atomic friction by a "minimal model" for thermally activated contact strengthening (Fig. 1). It reproduces the normal PT-like behavior of the friction force at sufficiently low and high temperatures. In the intermediate temperature range, the model explains naturally and in quantitative detail the experimentally observed possibility of a minimum and a maximum in the temperature dependence [Fig. 3(a)] and of a decreasing velocity dependence of the friction force [Fig. 4(b)].

We are grateful to André Schirmeisen for elucidating some experimental details of Refs. [4-7]. We thank the Deutsche Forschungsgemeinschaft (Grant No. RE 1344/7-1) and the ESF programs NATRIBO and FANAS (collaborative research project Nanoparma) for financial support. We thank the Deutsche Forschungsgemeinschaft (Grant No. RE 1344/7-1), the ESF programs NATRIBO and FANAS (collaborative research project Nanoparma), and the Open Access Publication Funds of Bielefeld University Library for financial support.
[1] F. P. Bowden and D. Tabor, The Friction and Lubrication of Solids (Oxford University Press, New York, 2001).

[2] Y. Sang, M. Dube, and M. Grant, Thermal Effects on Atomic Friction, Phys. Rev. Lett. 87, 17430 (2001); O. K. Dudko, A. E. Filippov, J. Klafter, and M. Urbakh, Dynamic Force Spectroscopy: A Fokker-Planck Approach, Chem. Phys. Lett. 352, 499 (2002); E. Riedo, E. Gnecco, R. Bennewitz, E. Meyer, and H. Brune, Interaction Potential and Hopping Dynamics Governing Sliding Friction, Phys. Rev. Lett. 91, 084502 (2003).

[3] L. Prandtl, Ein Gedankenmodell zur Kinetischen Theorie der Festen Körper, Z. Angew. Math. Mech. 8, 85 (1928); G. A. Tomlinson, A Molecular Theory of Friction, Philos. Mag. 7, 905 (1929).

[4] A. Schirmeisen, L. Jansen, H. Hölscher, and H. Fuchs, Temperature Dependence of Point Contact Friction on Silicon, Appl. Phys. Lett. 88, 123108 (2006).

[5] I. Barel, M. Urbakh, L. Jansen, and A. Schirmeisen, Multibond Dynamics of Nanoscale Friction: The Role of Temperature, Phys. Rev. Lett. 104, 066104 (2010); Temperature Dependence of Friction at the Nanoscale: When the Unexpected Turns Normal, Tribol. Lett. 39, 311 (2010).

[6] I. Barel, M. Urbakh, L. Jansen, and A. Schirmeisen, Unexpected Temperature and Velocity Dependencies of Atomic-Scale Stick-Slip Friction, Phys. Rev. B 84, 115417 (2011).

[7] L. Jansen, H. Hölscher, H. Fuchs, and A. Schirmeisen, Temperature Dependence of Atomic-Scale Stick-Slip Friction, Phys. Rev. Lett. 104, 256101 (2010).

[8] A. Vanossi, N. Manini, M. Urbakh, S. Zapperi, and E. Tosatti, Modeling Friction: From Nanoscale to Mesoscale, Rev. Mod. Phys. 85, 529 (2013).

[9] P. Hänggi, P. Talkner, and M. Borkovec, Reaction-Rate Theory: Fifty Years after Kramers, Rev. Mod. Phys. 62, 251 (1990).

[10] The generalized model from Ref. [6] also entails quite a number of additional fit parameters. As a caveat, we may furthermore remark that one of the energy barriers $\Delta E_{\text {on }}$ employed in Fig. 5 of Ref. [6] is much smaller than the thermal energy $k T$, and thus the rate approach itself becomes questionable [9].

[11] J.H. Dietrich, Time Dependent Friction and the Mechanics of Stick Slip, Pure Appl. Geophys. 116, 790 (1978); A. Ruina, Slip Instability and State Variable Friction Laws, J. Geophys. Res. 88, 10359 (1983).

[12] F. Heslot, T. Baumberger, B. Perrin, B. Caroli, and C. Caroli, Creep, Stick-Slip, and Dry-Friction Dynamics: Experiments and a Heuristic Model, Phys. Rev. E 49, 4973 (1994); B. N. J. Persson, Theory of Friction: Stress Domains, Relaxation, and Creep, Phys. Rev. B 51, 13568 (1995); Theory of Friction: Friction Dynamics for Boundary Lubricated Surfaces, Phys. Rev. B 55, 8004 (1997); O. M. Braun and J. Röder, Transition from StickSlip to Smooth Sliding: An Earthquakelike Model, Phys. Rev. Lett. 88, 096102 (2002); O. Ben-David, S. M. Rubinstein, and J. Fineberg, Slip-Stick and the Evolution of Frictional Strength, Nature (London) 463, 76 (2010).

[13] M. Evstigneev, L. Jansen, A. Schirmeisen, H. Fuchs, and P. Reimann, Contact Ageing in Atomic Friction, J. Phys. Condens. Matter 20, 354001 (2008). 
[14] Q. Li, T.E. Tullis, D. Goldsby, and R.W. Carpick, Frictional Ageing from Interfacial Bonding and the Origins of Rate and State Friction, Nature (London) 480, 233 (2011).

[15] Y. Liu and I. Szlufarska, Chemical Origins of Frictional Aging, Phys. Rev. Lett. 109, 186102 (2012).

[16] I. Barel, A. E. Filippov, and M. Urbakh, Formation and Rupture of Capillary Bridges in Atomic Scale Friction, J. Chem. Phys. 137, 164706 (2012).

[17] N. N. Gosvami, M. Feldmann, J. Peguiron, M. Moseler, A. Schirmeisen, and R. Bennewitz, Ageing of a Microscopic
Sliding Gold Contact at Low Temperatures, Phys. Rev. Lett. 107, 144303 (2011).

[18] W. K. Kim and M. L. Falk, Role of Intermediate States in Low-Velocity Friction between Amorphous Surfaces, Phys. Rev. B 84, 165422 (2011).

[19] M. Evstigneev and P. Reimann, Stick-Slip Statistics in Atomic Friction, Phys. Rev. B 87, 205441 (2013).

[20] E. Gnecco, R. Bennewitz, T. Gyalog, Ch. Loppacher, M. Bammerlin, E. Meyer, and H.-J. Güntherodt, Velocity Dependence of Atomic Friction, Phys. Rev. Lett. 84, 1172 (2000). 